Xi-Shi Tai and Yu-Pei Xia*

\title{
The crystal structure of $\left[\left(2,2^{\prime}\right.\right.$-bipyridine- $\left.k^{2} N, N\right)$ - bis(6-phenylpyridine-2-carboxylato- $\mathrm{k}^{2} \mathrm{~N}, \mathrm{O}$ ) cobalt(II)]-monohydrate, $\mathrm{C}_{36} \mathrm{H}_{26} \mathrm{~N}_{4} \mathrm{O}_{5} \mathrm{Co}$
}

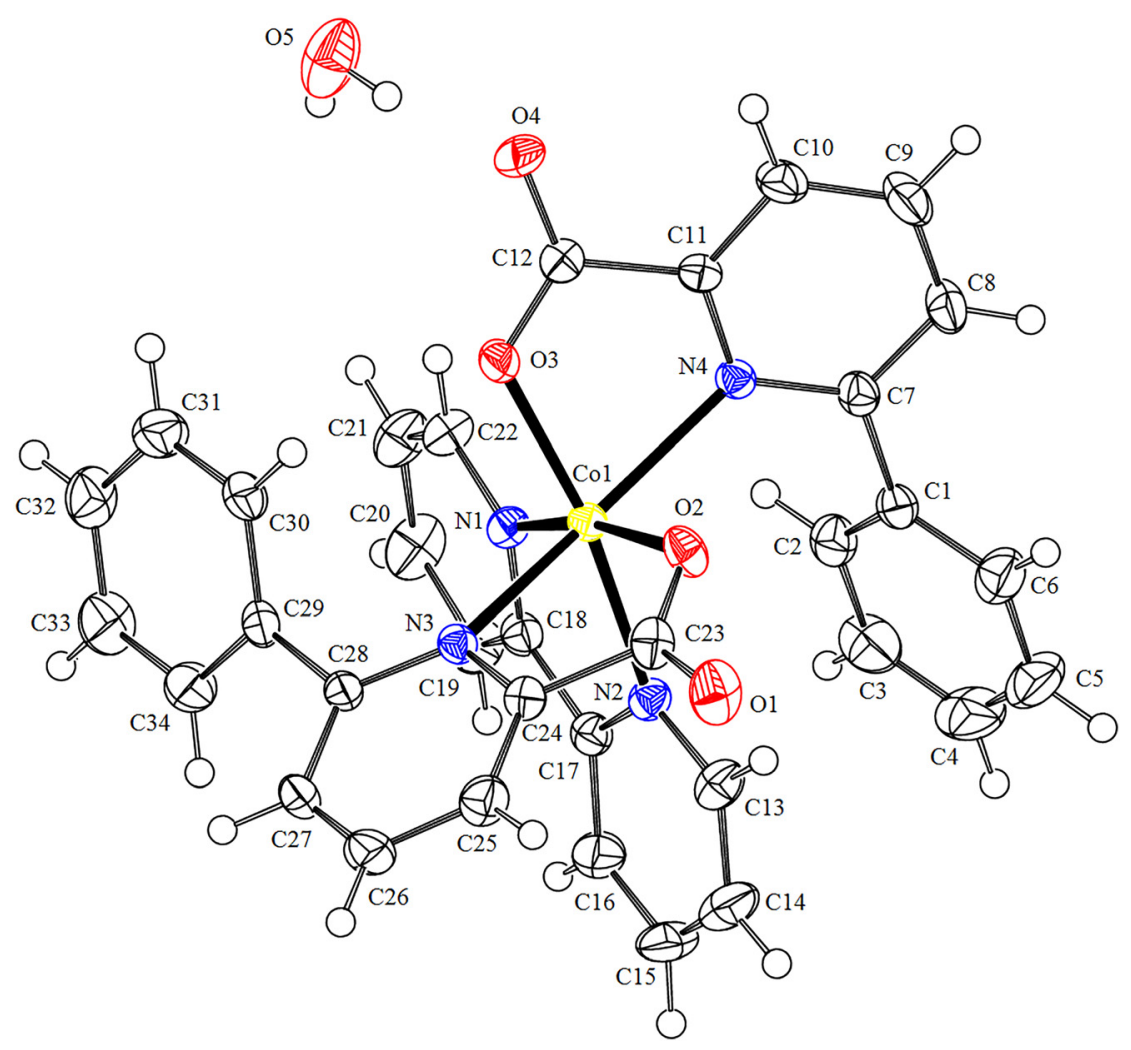

https://doi.org/10.1515/ncrs-2021-0473

Received December 14, 2021; accepted January 13, 2022; published online February 3, 2022

\section{Abstract}

$\mathrm{C}_{36} \mathrm{H}_{26} \mathrm{~N}_{4} \mathrm{O}_{5} \mathrm{Co}$, monoclinic, $\mathrm{C} 2 / c$ (no. 15), $a=29.3826(17) \AA$, $b=10.3153(5) \AA, c=19.9407(12) \AA, \beta=107.263(6)$, $V=5771.6(6) \AA^{3}, Z=8, R_{g t}(F)=0.0341, \mathrm{w} R_{r e f}\left(F^{2}\right)=0.0777$, $\mathrm{T}=200 \mathrm{~K}$.

CCDC no.: 2141869
Table 1: Data collection and handling.

\begin{tabular}{|c|c|}
\hline Crystal: & Red block \\
\hline Size & $0.14 \times 0.11 \times 0.09 \mathrm{~mm}$ \\
\hline Wavelength: & 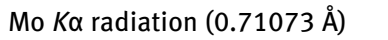 \\
\hline$\mu:$ & $0.65 \mathrm{~mm}^{-1}$ \\
\hline Diffractometer, scan mode: & SuperNova, $\omega$ \\
\hline$\theta_{\max }$, completeness: & $25.0^{\circ},>99 \%$ \\
\hline$N(h k l)_{\text {measured }}, N(h k l)_{\text {unique }}, R_{\text {int }}:$ & $11790,5081,0.025$ \\
\hline Criterion for $I_{\mathrm{obs}}, N(h k l)_{\mathrm{gt}}:$ & $I_{\text {obs }}>2 \sigma\left(I_{\text {obs }}\right), 4369$ \\
\hline$N(\text { param })_{\text {refined: }}$ & 400 \\
\hline Programs: & $\begin{array}{l}\text { Bruker [1], Olex2 [2], SHELX [3], } \\
\text { Diamond [4] }\end{array}$ \\
\hline
\end{tabular}

*Corresponding author: Xia Yu-Pei, College of Chemistry and Chemical Engineering, Weifang University, Weifang, Shandong 261061, P. R. China, E-mail: yopoxia@126.com

Xi-Shi Tai, College of Chemistry and Chemical Engineering, Weifang University, Weifang, Shandong 261061, P. R. China. https://orcid.org/ 0000-0002-0050-1900
The molecular structure is shown in the figure. Table 1 contains crystallographic data and Table 2 contains the list of the atoms including atomic coordinates and displacement parameters. 
Table 2: Fractional atomic coordinates and isotropic or equivalent isotropic displacement parameters $\left(\AA^{2}\right)$.

\begin{tabular}{|c|c|c|c|c|}
\hline Atom & $x$ & $y$ & $z$ & $U_{\text {iso }}{ }^{*} / U_{\text {eq }}$ \\
\hline Co1 & $0.37802(2)$ & $0.42972(2)$ & $0.30897(2)$ & $0.01825(9)$ \\
\hline 01 & 0.36059 (5) & $0.49317(16)$ & $0.49866(8)$ & $0.0372(4)$ \\
\hline 02 & 0.38739 & $0.50687(14)$ & $0.40542(7)$ & $0.0266(3)$ \\
\hline 03 & $0.36283(5)$ & $0.59292(13)$ & $0.24949(7)$ & $0.0262(3)$ \\
\hline 04 & $0.39226(5)$ & 0.77367 (13) & $0.21700(8)$ & $0.0293(4)$ \\
\hline N1 & $0.37359(6)$ & $0.30813(15)$ & $0.22105(8)$ & $0.0211(4)$ \\
\hline $\mathrm{N} 2$ & $0.38644(6)$ & $0.23334(16)$ & $0.35189(9)$ & 0.0214 (4) \\
\hline N3 & 0.30505 & $0.41056(15)$ & $0.32057(8)$ & 0.0189 (4) \\
\hline N4 & $0.45040(5)$ & $0.51432(15)$ & $0.32317(8)$ & $0.0191(4)$ \\
\hline $\mathrm{C} 1$ & $0.50075(7)$ & $0.3418(2)$ & $0.38825(11)$ & $0.0247(5)$ \\
\hline $\mathrm{C} 2$ & $0.48848(7)$ & $0.2465(2)$ & $0.33719(12)$ & $0.0304(5)$ \\
\hline $\mathrm{H} 2$ & 0.474744 & 0.269763 & 0.290478 & $0.036^{*}$ \\
\hline $\mathrm{C} 3$ & $0.49644(8)$ & $0.1168(2)$ & $0.35490(15)$ & $0.0434(6)$ \\
\hline $\mathrm{H} 3$ & 0.488338 & 0.053396 & 0.320224 & $0.052^{*}$ \\
\hline $\mathrm{C} 4$ & $0.51642(9)$ & $0.0824(3)$ & $0.42418(17)$ & $0.0530(8)$ \\
\hline $\mathrm{H} 4$ & 0.521417 & -0.004634 & 0.436416 & $0.064^{*}$ \\
\hline $\mathrm{C} 5$ & $0.52905(9)$ & $0.1767(3)$ & $0.47548(15)$ & $0.0493(7)$ \\
\hline H5 & 0.542441 & 0.152782 & 0.522164 & 0.059 * \\
\hline C6 & $0.52194(8)$ & $0.3065(2)$ & $0.45799(12)$ & $0.0369(6)$ \\
\hline $\mathrm{H} 6$ & 0.531252 & 0.369776 & 0.492596 & $0.044^{*}$ \\
\hline $\mathrm{C} 7$ & $0.49307(7)$ & $0.4804(2)$ & $0.36802(10)$ & $0.0224(4)$ \\
\hline $\mathrm{C} 8$ & $0.52907(7)$ & $0.5707(2)$ & $0.39357(12)$ & $0.0304(5)$ \\
\hline $\mathrm{H} 8$ & 0.557729 & 0.545708 & 0.425621 & $0.036^{*}$ \\
\hline $\mathrm{C9}$ & $0.52217(8)$ & $0.6974(2)$ & $0.37129(13)$ & $0.0356(6)$ \\
\hline H9 & 0.545932 & 0.758954 & 0.388162 & $0.043^{*}$ \\
\hline C10 & $0.47936(7)$ & $0.7313(2)$ & $0.32344(12)$ & $0.0297(5)$ \\
\hline $\mathrm{H} 10$ & 0.474130 & 0.815590 & 0.306322 & $0.036^{*}$ \\
\hline $\mathrm{C} 11$ & $0.44455(7)$ & $0.63836(19)$ & $0.30150(10)$ & $0.0196(4)$ \\
\hline $\mathrm{C} 12$ & $0.39626(7)$ & 0.67217 (19) & $0.25174(10)$ & 0.0209 (4) \\
\hline C13 & $0.39173(8)$ & $0.2008(2)$ & $0.41865(11)$ & $0.0291(5)$ \\
\hline $\mathrm{H} 13$ & 0.400681 & 0.264945 & 0.452849 & $0.035^{*}$ \\
\hline C14 & $0.38456(9)$ & $0.0769(2)$ & $0.43926(13)$ & $0.0383(6)$ \\
\hline $\mathrm{H} 14$ & 0.387819 & 0.058613 & 0.486116 & $0.046^{*}$ \\
\hline C15 & $0.37257(8)$ & $-0.0185(2)$ & 0.38965 (13) & $0.0408(6)$ \\
\hline $\mathrm{H} 15$ & 0.367103 & -0.102612 & 0.402189 & $0.049^{*}$ \\
\hline C16 & $0.36870(8)$ & $0.0111(2)$ & $0.32107(12)$ & $0.0330(5)$ \\
\hline $\mathrm{H} 16$ & 0.361428 & -0.053126 & 0.286811 & 0.040 * \\
\hline C17 & $0.37575(7)$ & 0.13793 (19) & $0.30349(10)$ & $0.0220(5)$ \\
\hline C18 & $0.37301(7)$ & $0.17853(19)$ & $0.23139(10)$ & $0.0221(5)$ \\
\hline C19 & $0.37138(8)$ & $0.0910(2)$ & $0.17826(12)$ & $0.0320(5)$ \\
\hline $\mathrm{H} 19$ & 0.371103 & 0.002305 & 0.186486 & $0.038^{*}$ \\
\hline $\mathrm{C} 20$ & $0.37017(9)$ & $0.1372(2)$ & $0.11292(12)$ & $0.0387(6)$ \\
\hline $\mathrm{H} 2 \mathrm{O}$ & 0.369031 & 0.079774 & 0.076497 & $0.046^{*}$ \\
\hline $\mathrm{C} 21$ & $0.37066(8)$ & $0.2687(2)$ & $0.10190(12)$ & $0.0357(6)$ \\
\hline $\mathrm{H} 21$ & 0.369960 & 0.301582 & 0.058186 & $0.043^{*}$ \\
\hline $\mathrm{C} 22$ & $0.37220(7)$ & $0.3504(2)$ & $0.15700(11)$ & $0.0273(5)$ \\
\hline $\mathrm{H} 22$ & 0.372284 & 0.439339 & 0.149412 & $0.033^{*}$ \\
\hline $\mathrm{C} 23$ & $0.35611(7)$ & $0.4801(2)$ & $0.43545(11)$ & $0.0247(5)$ \\
\hline $\mathrm{C} 24$ & $0.30949(7)$ & $0.42413(19)$ & $0.38934(10)$ & 0.0205 (4) \\
\hline $\mathrm{C} 25$ & $0.27426(7)$ & $0.3887(2)$ & $0.41862(12)$ & $0.0301(5)$ \\
\hline $\mathrm{H} 25$ & 0.278336 & 0.401460 & 0.466212 & $0.036^{*}$ \\
\hline $\mathrm{C} 26$ & $0.23288(7)$ & $0.3339(2)$ & $0.37609(12)$ & $0.0335(5)$ \\
\hline $\mathrm{H} 26$ & 0.208918 & 0.306882 & 0.394631 & $0.040^{*}$ \\
\hline $\mathrm{C} 27$ & $0.22788(7)$ & $0.3203(2)$ & $0.30573(12)$ & $0.0285(5)$ \\
\hline $\mathrm{H} 27$ & 0.200326 & 0.283316 & 0.276225 & $0.034^{*}$ \\
\hline
\end{tabular}

Table 2: (continued)

\begin{tabular}{lrrrr}
\hline Atom & $\boldsymbol{x}$ & $\boldsymbol{y}$ & $\boldsymbol{z}$ & $\boldsymbol{U}_{\text {iso }}{ }^{*} \boldsymbol{U}_{\text {eq }}$ \\
\hline C28 & $0.26386(7)$ & $0.36166(18)$ & $0.27835(11)$ & $0.0213(4)$ \\
C29 & $0.25562(7)$ & $0.36050(19)$ & $0.20145(11)$ & $0.0229(5)$ \\
C30 & $0.26489(7)$ & $0.4712(2)$ & $0.16832(11)$ & $0.0278(5)$ \\
H30 & 0.278860 & 0.542675 & 0.195011 & $0.033^{*}$ \\
C31 & $0.25359(8)$ & $0.4764(2)$ & $0.09619(12)$ & $0.0369(6)$ \\
H31 & 0.259664 & 0.551339 & 0.074406 & $0.044^{*}$ \\
C32 & $0.23323(8)$ & $0.3703(2)$ & $0.05616(12)$ & $0.0382(6)$ \\
H32 & 0.225604 & 0.373781 & 0.007473 & $0.046^{*}$ \\
C33 & $0.22433(9)$ & $0.2600(2)$ & $0.08832(13)$ & $0.0415(6)$ \\
H33 & 0.211018 & 0.188257 & 0.061373 & $0.050^{\star}$ \\
C34 & $0.23500(8)$ & $0.2546(2)$ & $0.16062(12)$ & $0.0345(6)$ \\
H34 & 0.228338 & 0.179814 & 0.182007 & $0.041^{*}$ \\
O5 & $0.36053(7)$ & $0.7566(2)$ & $0.06873(9)$ & $0.0535(5)$ \\
H5A & 0.369542 & 0.750866 & 0.113268 & $0.080^{*}$ \\
H5B & 0.359665 & 0.678614 & 0.054576 & $0.080^{*}$ \\
\hline
\end{tabular}

\section{Source of material}

The title compound was synthesized by the one-pot method according to the following procedure: 6-pheny lpyridine-2-carboxylic acid (0.0996 g, $0.5 \mathrm{mmol}), \mathrm{NaOH}$ $(0.020 \mathrm{~g}, \quad 0.5 \mathrm{mmol}), \mathrm{Co}\left(\mathrm{CH}_{3} \mathrm{COO}\right)_{2} \cdot 4 \mathrm{H}_{2} \mathrm{O}(0.1245 \mathrm{~g}$, $0.5 \mathrm{mmol})$, and 2,2'-bipyridine $(0.078 \mathrm{~g}, 0.5 \mathrm{mmol})$ were dissolved in a mixed solvent of $20 \mathrm{~mL}$ of water and ethanol ( $v: v=1: 1)$ at room temperature. The solution immediately turns light amaranth. The reaction mixture then continued to react with stirring for $4.5 \mathrm{~h}$ at $75{ }^{\circ} \mathrm{C}$. Then the reaction mixture was cooled to room temperature and filtered. The light red crystals of the title compound were obtained from the above filtrate after 10 days.

\section{Experimental details}

Coordinates of hydrogen atoms were refined using different models. The hydrogen atoms were positioned geometrically $\left(\mathrm{C}-\mathrm{H}=0.93 \AA\right.$ and $\mathrm{O}-\mathrm{H}=0.85 \AA$ ). Their $U_{\text {iso }}$ values were set to $1.2 U_{\text {eq }}$ or $1.5 U_{\text {eq }}$ of the parent atoms.

\section{Comment}

Many studies have shown that some metal complexes show excellent potential applications [5]. As an important class of metal complexes, cobalt(II) complexes have also especially shown excellent properties in many aspects such as catalytic for the electrochemical reduction of $\mathrm{CO}_{2}$, DNA binding and antibacterial activity, magnetic property and redox property, 
large zero-field splittings, and single-ion magnets [6-8]. $2,2^{\prime}$-Bipyridine plays a significant role as a second ligand in many complexes [9]. Some complexes of $\mathrm{Mn}$ (II), $\mathrm{Pb}$ (II), $\mathrm{Cu}$ (II), $\mathrm{Zn}$ (II) and Co(II) have been synthesized and structural characterized with 6-phenylpyridine-2-carboxylate as an excellent ligand in our group [10-14]. In this paper, we present a new $\mathrm{Co}(\mathrm{II})$ complex, which was synthesized using $\mathrm{Co}\left(\mathrm{CH}_{3} \mathrm{COO}\right)_{2}$. $4 \mathrm{H}_{2} \mathrm{O}, 2,2$-bipyridine, 6-phenylpyridine-2-carboxylic acid, and $\mathrm{NaOH}$.

Analysis of the crystal structure data shows that the $\mathrm{Co}(\mathrm{II})$ complex contains a $\mathrm{Co}(\mathrm{II})$ ion, two 6-phenylpyridine2-carboxylate ligands, a 2,2'-bipyridine ligand, and a lattice water molecule (see the Figure). The Co(II) ion adopts a distorted octahedral coordination mode by coordinating with two carboxylate oxygen atoms (02 and $\mathrm{O3}$ ), two pyridine nitrogen atoms ( $\mathrm{N} 3$ and $\mathrm{N} 4$ ) of two different 6-phenylpyridine-2-carboxylate ligands and two nitrogen atoms (N2 and N1) of one 2,2'-bipyridine ligand. The bond angles around $\mathrm{Co}(\mathrm{II})$ are $101.97(6)^{\circ}(\mathrm{N} 1-\mathrm{Co} 1-\mathrm{N} 3), 78.48(6)^{\circ}$ $(\mathrm{O} 2-\mathrm{Co} 1-\mathrm{N} 3), 82.54(6)^{\circ}(\mathrm{O} 2-\mathrm{Co} 1-\mathrm{N} 4)$ and $99.06(6)^{\circ}(\mathrm{N} 1-$ Co1-N4), respectively, indicating N1, N3, N4 and $\mathrm{O} 2$ atoms are at the equatorial plane. And the bond angle of N2-Co1$\mathrm{O} 3$ is $167.71(6)^{\circ}$, showing $\mathrm{N} 2$ and $\mathrm{O} 3$ atoms are in the axial position. The Co-N distances fall in the range of 2.1280(16) $2.2378(15)^{\circ} \mathrm{A}$ (Co1-N1), 2.1280(16) ${ }^{\circ} \AA$, (Co1-N2), 2.1844(16) $)^{\circ}$, (Co1-N3), 2.2325(16) ${ }^{\circ} \AA$, Co1-N4, 2.2378(15) ${ }^{\circ} \AA$ and the Co-O distances are $2.0233(14)^{\circ} \AA$ (Co1-O2) and 2.0313(14) ${ }^{\circ} \AA$ (Co1-03), respectively. The dihedral angles between of ring 1 (C1-C2-C3-C4-C5-C6) and ring 2 (C7-C8-C9-C10-C11N4), ring 3 (C29-C30-C31-C32-C33-C34) and ring 4 (C24$\mathrm{C} 25-\mathrm{C} 26-\mathrm{C} 27-\mathrm{C} 28-\mathrm{N} 3)$ are $133.4^{\circ}$ and $132.4^{\circ}$, respectively, which indicates that each 6-phenylpyridine-2-carboxylate ligand is not coplanar. The complex molecules form 1D chained structure through hydrogen bonds between the water molecule and the 6-phenylpyridine-2-carboxylate ligand.

Author contributions: All the authors have accepted responsibility for the entire content of this submitted manuscript and approved submission.

Research funding: This project was supported by the National Natural Science Foundation of China (No. 21171132), the Natural Science Foundation of Shandong (ZR2014BL003), the Project of Shandong Province Higher Educational Science and Technology Program (J14LC01) and Science Foundation of Weifang.

Conflict of interest statement: The authors declare no conflicts of interest regarding this article.

\section{References}

1. Bruker. SAINT and SADABS; Bruker AXS Inc.: Madison, Wisconsin, USA, 2000.

2. Dolomanov O. V., Bourhis L. J., Gildea R. J., Howard J. A. K., Puschmann H. olex2: a complete structure solution, refinement and analysis program. J. Appl. Crystallogr. 2009, 42, 339-341.

3. Sheldrick G. M. Crystal structure refinement with SHELXL. Acta Crystallogr. 2015, C71, 3-8.

4. Brandenburg K. DIAMOND. Visual Crystal Structure Information System. Ver. 3.2; Crystal Impact: Bonn, Germany, 2012.

5. Shyam P., Ravi K.-V., Srinivas G., Navaneetha N., Venugopal R.-K., Satyanarayana S. Influence of Co(III) polypyridyl complexes on luminescence behavior, DNA binding, photocleavage, antimicrobial activity and molecular docking studies. J. Fluoresc. 2021, 31, 1009-1021.

6. Nganga J., Chaudhri N., Brckner C., Angeles-Boza A.-M. $\beta-$ oxochlorin cobalt(II) complexes catalyze the electrochemical reduction of $\mathrm{CO}_{2}$. Chem. Commun. 2021, 57, 4396-4399.

7. Vinayak A., Basappa C.-Y., Vinutha K., Krishna P.-M. Characterization studies of novel series of cobalt(II), nickel(II) and copper(II) complexes: DNA binding and antibacterial activity. J. Pharmaceut. Invest. 2021, 51, 347-359.

8. Bamberger H., Albold U., Midlíková J.-D., Su C.-Y., Deibel N., Hunger D., Hallmen1 P.-P., Neugebauer P., Beerhues J., Demeshko S., Meyer F., Sarkar B., Slageren J.-V. Iron(II), cobalt(II), and nickel(II) complexes of bis(sulfonamido)benzenes: redox properties, large zero-field splittings, and single-ion magnets. Inorg. Chem. 2021, 60, 2953-2963.

9. Helen O.-O., Abiodun O.-E., Raymond A.-A. Mixed ligand complexes of copper(II) with benzoyltrifluoroacetone, 1,10-phenanthroline and 2,2'-bipyridine: structure, spectroscopic and antimicrobial properties. J. Mol. Struct. 2021, 1246, 131826.

10. Tai X.-S., Liang L., Li X. T., Cao S. H., Wang L.-H. Crystal structure of diaqua-bis $\left(\mu_{2}-6\right.$-phenylpyridine-2-carboxylate- $\left.\kappa^{3} N, O: O\right)$ bis(6-phenylpyridine-2-carboxylato- $\kappa^{2} N, O$ )lead(II) - N,Ndimethylformamide - water (1/2/4), $\mathrm{C}_{54} \mathrm{H}_{58} \mathrm{~N}_{6} \mathrm{O}_{16} \mathrm{~Pb}_{2}$. Z. Kristallogr. N. Cryst. Struct. 2021, 236, 1199-1201.

11. Tai X.-S., Wang Z.-J., Ouyang J., Li Y.-F., Zhang W., Jia W.-L., Wang L.-H. The crystal structure of [(phenantroline- $\left.\kappa^{2} N, N^{\prime}\right)$ bis(6-phenylpyridine-2-carboxylate- $\kappa^{2} N, O$ ) cobalt(II)] monohydrate, $\mathrm{C}_{36} \mathrm{H}_{26} \mathrm{~N}_{4} \mathrm{O}_{5}$ Co. Z. Kristallogr. N. Cryst. Struct. 2021, 236, 1309-1311.

12. Wang L.-H., Liang L., Li X.-T., Cao S.-H., Tai X.-S. The crystal structure of bis(6-phenylpyridine- 2-carboxylato- $\kappa^{2} \mathrm{~N}, \mathrm{O}$ ) copper(II), $\mathrm{C}_{24} \mathrm{H}_{16} \mathrm{~N}_{2} \mathrm{OC}$. Z. Kristallogr. N. Cryst. Struct. 2021, 236, 1251-1253.

13. Wang L.-H., Wang Z.-J., Ouyang J., Tai X.-S. The crystal structure of bis(6-phenylpyridine- 2-carboxylate- $N, O$ )-(2,2-bipyridine- $N$,) zinc(II) monohydrate, $\mathrm{C}_{34} \mathrm{H}_{26} \mathrm{~N}_{4} \mathrm{O}_{5} \mathrm{Zn}$. Z. Kristallogr. N. Cryst. Struct. 2021, 236, 1297-1299.

14. Tai X.-S., Zhang L.-L., Liu L.-L., Cao S.-H., Wang L.-H. The crystal structure of bis(ethanol-kO)- bis(6-aminopicolinato- $N, O$ ) manganese(II), $\mathrm{C}_{16} \mathrm{H}_{22} \mathrm{O}_{6} \mathrm{~N}_{4} M n$. Z. Kristallogr. N. Cryst. Struct. 2021, 236, 551-552. 